\title{
Zurück zum Pre Injury Level nach Verletzungen der unteren Extremität - eine Einteilung funktioneller Assessments
}

Von Return to Activity zu Return to Competition ist es für Sportler nach einer Verletzung ein weiter Weg. Die Post Injury Pyramide stellt die einzelnen Rehabilitationsphasen auf diesem Weg hierarchisch dar. Um Sportler adäquat vorzubereiten, arbeiten Therapeuten und Trainer mit funktionellen Assessments und Trainingsreizen, die den jeweiligen Phasen der Post Injury Pyramide entsprechen.

Der Weg zurück zum Sport und die damit verknüpften Entscheidungen sind komplex. Funktionelle Assessments helfen, diesen Prozess zu objektivieren und ihn begründbar zu steuern, und motivieren Patienten und Therapeuten gleichermaßen, zielorientiert zu arbeiten.

Die Rückkehr zum Sport ist ein individueller Prozess und von der Zielsetzung und der Teilhabe des Patienten abhängig. Für den Therapeuten ist es notwendig, die persönliche Zielsetzung des Patienten zu kennen und einzuschätzen. Erst dann kann eine systematische und konkrete Planung für die Rehabilitation erstellt werden. Funktionelle Assessments begleiten die Rehabilitation, um Defizite aufzudecken und den Verlauf zu protokollieren. Dieser Ansatz entspricht den Empfehlungen der WHO, das Gesundheitsproblem eines Patienten auf Grundlage der ICF Klassifikation zu betrachten [29]. Traditionelle Nachbehandlungsschemata, die sich ausschließlich an Zeitvorgaben orientieren, treten immer mehr in den Hintergrund, da sie keine Vorhersagen bezüglich einer Rückkehr zu Sport und Alltagsbelastung, vor allem unmittelbar nach einer Operation, erlauben [13, 15].

:: Die persönliche Zielsetzung des Patienten zu kennen, ist für die Planung der Rehabilitation essenziell.

\section{Differenzierung der Begrifflichkeiten}

Wenn Patienten nach einer Verletzung wieder in der Lage sind, sich der gleichen sportlichen Belastung wie vor der Verletzung auszusetzen, spricht man von einem erreichten Pre Injury Level [9]. Verschiedene Arbeitsgruppen haben Einteilungen vorgenommen, die sich an dem Grad der Aktivität und der sportlichen Belastung orientieren $[6,26]$. Diese Einteilungen können bei der Ein- stufung und Subgruppenbildung hilfreich sein, um Patienten auf ihrem Weg zurück zu Alltag und Sport einzuschätzen.

Sucht man zu dieser Thematik in der Literatur, so wird man auf verschiedene Begrifflichkeiten aufmerksam, die von den jeweiligen Experten unterschiedlich definiert werden. Eine einheitliche Darstellung existiert nicht. Grundsätzlich lassen sich die verwendeten Begrifflichkeiten hierarchisch darstellen ( $\bullet$ Abb. 1).

\section{Return to Activity}

Return to Activity bedeutet, dass der Sportler Basisfunktionen wie den Einbeinstand, die Kniebeuge oder diverse Sprünge wieder problemlos beherrscht. Diese Fähigkeiten gelten als Mindestvoraussetzung, um die entsprechende Sportart wieder ausüben oder einer bestimmten Tätigkeit wieder nachgehen zu können.

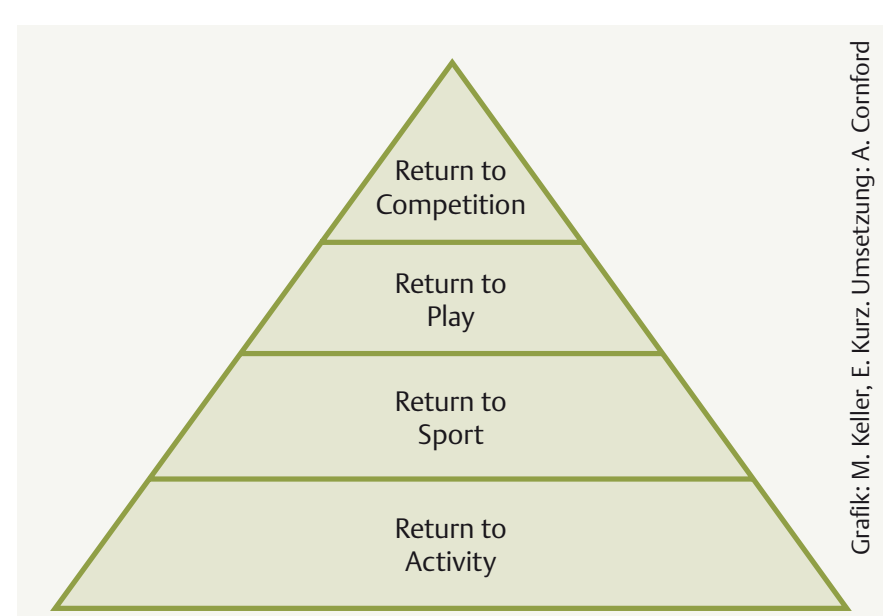

Abb. 1 Post Injury Pyramide 
Zeigen sich bei diesen Funktionen Dysbalancen oder Asymmetrien, ist eine Wiedereingliederung in den Sport oder die Alltagsbelastung nicht uneingeschränkt zu empfehlen [14].

\section{Return to Sport}

Return to Sport ist dann erreicht, wenn der Sportler wieder sportartspezifisch trainieren kann. Dieses Niveau kann ein Sportler am Ende der Rehabilitation erlangen. Die Übungen sollten in dieser Phase sportartspezifischer werden und nach Möglichkeit in der gewohnten Trainingsumgebung stattfinden. Diesen Teil der Rehabilitation beschreibt man auch als „On Field Rehaibilitation“ [7]. Dabei werden Situationen simuliert, die bei der Ausübung der Sportart auftreten können. Beispielsweise trainieren Fußballer dann schnelle Richtungswechsel und Start-Stopp-Bewegungen. Diese Phase wird im Mannschaftssport auch als „Return to reduced Team Training" bezeichnet und zeichnet sich dadurch aus, dass bei den Übungen noch kein Körperkontakt stattfindet [4]. Denkbar ist auch, dass der Sportler zunächst nur teilweise am Mannschaftstraining teilnimmt. Selbst wenn eine Rehabilitation bis dahin ohne negative Reaktionen verlaufen ist, bleibt es noch unklar, ob der Athlet sein Wettkampfniveau wieder erreichen wird.

\section{Return to Play}

Unter Return to Play versteht man die Rückkehr zur vollen Sportfähigkeit [20,22]. Der Sportler ist entsprechend seiner Funktion wiederhergestellt und gesund, hat keine posttraumatischen Einschränkungen mehr und kann beispielsweise bei Teamsportarten uneingeschränkt am gesamten Mannschaftstraining teilnehmen. Übungen mit Körperkontakt oder Aktionen wie Pressschlag oder Tackling sollten auf diesem Niveau im Training simuliert werden. Diese Phase wird auch als „Return to full Team Training“ bezeichnet [4]. Offen bleibt, ob der Athlet eine ausreichende Fitness aufgebaut hat, um über die volle Dauer eines Fußballspiels oder die gesamte Zeit eines Tennismatches seine Leistung abzurufen. Daher ist es wichtig, dass er gezielt an seiner sportartspezifischen Fitness arbeitet und sich beim Training die nötige Sicherheit für den Wettkampf holt. Erst wenn der Athlet dieses Niveau ohne negative Reaktion über einen Zeitraum von circa zwei Wochen halten kann, ist über eine Teilnahme am Wettkampf zu entscheiden [20].

Die Arbeitsgruppe um Ardern untersuchte in ihrer Übersichtsarbeit, welches Pre Injury Level Patienten nach einer Verletzung des vorderen Kreuzbandes erreichen können. In 48 Studien wurden insgesamt 5770 Patienten evaluiert. Dabei stellten Ardern et al. fest, dass 82 Prozent der Patienten nach ihrer Verletzung zwar wieder einen Sport ausüben konnten, aber nur 44 Prozent ihr Wettkampfniveau wieder erreichten [1]. Eine Differenzierung zwischen dem Ausführen einer Sportart und dem Ausüben eines Sports auf Leistungs- oder Wettkampfniveau ist daher notwendig.

\section{Return to Competition}

Kann ein Sportler wieder über die gesamte Dauer oder den gesamten Umfang am Wettkampf teilnehmen, wird dies als Return to Competition oder „Return to competitive Match“ bezeichnet [4, 5]. Der verantwortliche Trainer traut dem Athleten nach seiner
Verletzung zu, eine physische und psychische Leistung zu erbringen, die dem Wettbewerb standhalten kann.

\section{Funktionelle Assessments als Entscheidungshilfe}

Bereits in den 90er-Jahren wurden Sprungtests eingesetzt, um die dynamische Belastbarkeit von Patienten zu evaluieren. Die wohl bekannteste Testbatterie aus dieser Zeit besteht aus vier verschiedenen Sprungtests und vergleicht die betroffene mit der nicht betroffenen Extremität [2, 19]. Aber auch andere Arbeitsgruppen nutzten Sprungtests als Entscheidungshilfe für eine Rückkehr zum Sport [10, 18, 21]. Der Limb Symmetry Index (LSI) dient bei diesen funktionellen Assessments dazu, eine Seitensymmetrie zu quantifizieren und messbar zu machen (S. 14).

Isolierte Funktionsprüfungen wie Kraft- oder Beweglichkeitsmessungen werden eingesetzt, um Faktoren zu identifizieren, die mit einem erhöhten Verletzungsrisiko assoziiert sind [16], und um Erfolge im Rehabilitationsverlauf zu dokumentieren [12].

Um komplexere Fähigkeiten zu prüfen, arbeitet man mit „On Field Tests“ [7] oder mit einer komplexen Trainingssteuerung, die eine systematische Belastungssteigerung beinhaltet [4]. Dabei werden vor allem Eigenschaften wie Agilität, Schnelligkeit, komplexe Kraft und Ausdauer geprüft. Diese unterschiedlichen Assessments können den einzelnen Ebenen der Post Injury Pyramide zugeordnet werden und müssen vor allem beim Return to Play nach dem Anforderungsprofil der jeweiligen Sportart ausgewählt werden ( Tab. 1). Der Zeitpunkt des Return to Competition lässt sich nur schwer mit Tests simulieren und fällt in den Kompetenz-

Tab. 1 Exemplarische Zuordnung funktioneller Assessments zu Ebenen der Post Injury Pyramide für die Sportart Fußball

\begin{tabular}{|c|c|}
\hline Post Injury Ebene & Assessments \\
\hline Return to Activity & 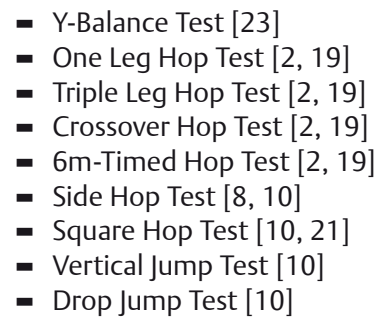 \\
\hline Return to Sport & $\begin{array}{l}\text { - } \text { Figure-of-8 Test }[8] \\
\text { - Hexagon Test }[25,30] \\
\text { - } \text { Up-Down Hop Test }[8] \\
\text { - } \text { Tapping Test [11] }\end{array}$ \\
\hline Return to Play & $\begin{array}{l}\text { - T-Test [25] } \\
\text { - Münchner Dreieck Test [25] } \\
\text { - } \text { Illinois Agility Test [25] } \\
\text { - Zig-Zag Test [25] } \\
\text { - } 505 \text { Agility Test [25] } \\
\text { - } \text { Yo-Yo Test [3] }\end{array}$ \\
\hline
\end{tabular}


bereich eines Trainerstabs. Eine interdisziplinäre Entscheidungsfindung ist dabei allerdings anzustreben.

\section{Progressive Belastungssteuerung als Entscheidungshilfe}

Gerade am Ende der Rehabilitation in den Phasen Return to Sport, Return to Play und Return to Competition wird es immer schwieriger, spezifische Tests und standardisierte Assessments als Entscheidungshilfe für die Rückkehr zu Belastungen zu finden, welche auch die Zielfunktion und die sportartspezifischen Reize simulieren. Daher kommt in der Praxis häufig die Methode „Versuch und Irrtum" zum Einsatz. Dabei setzt man in einer Trainingseinheit Belastungsreize und beobachtet, ob eine Reaktion auf diese Belastung folgt. Eine genaue Dokumentation ist hier zwingend erforderlich. Therapeuten oder Trainer halten die genaue Dosierung der Trainingseinheit fest, indem sie beispielsweise alle dynamischen Kontakte zählen. Gibt der Athlet bei der Besprechung vor der nächsten Einheit ein positives Feedback, kann die Belastung Schritt für Schritt weiter erhöht werden. Zeigen sich negative Reaktionen, die auf die Belastung zurückzuführen sind, sollten die Trainingsreize reduziert oder zumindest nicht weiter erhöht werden. Hier empfiehlt es sich, die 24-Stunden-Regel anzuwenden. Laut dieser Regel dürfen die Strukturen nach einem neuen oder erhöhten Trainingsreiz zwar durchaus reagieren, allerdings sollte diese Reaktion nach 24 Stunden wieder vollständig abgeklungen sein [24]. Das Training in einer späten Rehabilitationsphase ist immer eine minimale und geplante Überlastung. Nur so lässt sich die Belastbarkeit der verletzten Struktur auf das erforderliche Belastungsniveau anheben.

: Durch die Strategie „Versuch und Irrtum“ nach der 24-Stunden-Regel lässt sich ein adäquater Trainingsreiz

in späten Rehabilitationsphasen steuern.

In verschiedenen Einrichtungen wird dieses progressive Vorgehen in Phasen beschrieben. Jeder Phase sind Trainingsreize und Trainingsinhalte zugeordnet. Erst wenn eine Phase erfolgreich abgeschlossen ist, dürfen die Inhalte aus der nächsten Phase für die Trainingssteuerung ausgewählt werden. Diese phasenbasierten Trainingskonzepte wurden unter anderem für Fußball, American Football, Basketball und den Alpinen Skisport beschrieben [4, 17, $27,28]$.
Mehrere Arbeitsgruppen beschäftigen sich mit der Rückkehr zum Sport nach Verletzungen an der unteren Extremität. Eine einheitliche Vorgehensweise gibt es derzeit noch nicht. Es zeichnet sich jedoch ab, dass Tests, welche die Funktionen prüfen und sportartspezifische Belastungen simulieren, als Entscheidungshilfe für eine sichere Rückkehr zum Sport hilfreich sind. Die Auswahl der Tests sollte progressiv und der Wundheilung angepasst sein. Welche Tests tatsächlich in der Lage sind, Retraumatisierungen vorherzusagen, muss weiter untersucht werden. Für die Praxis eignen sich Testbatterien, die einfach umzusetzen und kostengünstig sind, damit diese auch flächendeckend in der Rehabilitation eingesetzt werden. Eine einheitliche Definition der verschiedenen Begrifflichkeiten wäre in Zukunft wünschenswert.

Literaturverzeichnis am Ende der HTML-Version unter: www.thieme-connect.de/products/manuelletherapie

\section{AUTOREN}

Matthias Keller, Physiotherapeut, B. A. leitet zusammen mit Oliver Schmidtlein das OS Institut -Bewegung für Orthopädie und Sportmedizin - und ist bei OSPHYSIO als Physiotherapeut tätig. Er ist Dozent für medizinische Trainingstherapie und Mitglied der Expertengruppe „Prävention, konservative Therapie und Rehabilitation“ der Deutschen Kniegesellschaft e.V.

Matthias Keller, B.A.

OS Institut

Bewegung für Orthopädie und Sportmedizin

Schmidtlein \& Keller GbR

Balanstr. 73/Haus 8

81541 München

mk@osinstitut.de

www.osinstitut.de

Eduard Kurz, Diplom-Sportwissenschaftler, Sporttherapeut und Athletiktrainer, ist wissenschaftlicher Mitarbeiter am Universitätsklinikum in Jena und verantwortlich für die Aus- und Fortbildung im Thüringer Behinderten- und Rehabilitationssport-Verband. Am OS Institut ist er Dozent und Leiter des Forschungsbereichs.

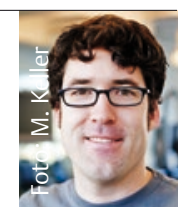

manuelletherapie 2016; 20: 16-18

(c) Georg Thieme Verlag KG

Stuttgart · New York · ISSN 1433-2671 